\title{
Observation of Recorded Marks of MO Disk by Scanning Near-Field Magneto-Optical Microscope
}

\author{
K. Sato, T. Ishibashi, T. Yoshida, J. Yamamoto, A. Iijima, Y. Mitsuoka*, and K. Nakajima* \\ Faculty of Technology, Tokyo University of Agriculture and Technology, Koganei, Tokyo 184-8588, Japan \\ *Technology Center, Seiko Instruments Inc, Matsudo, Chiba 270-2222, Japan
}

A scanning near-field magneto-optical microscope using a bent-type fiber probe operating as an AFM cantilever has been investigated for magneto-optical imaging. Using a polarization modulation technique, the signal to noise ratio as well as the contrast ratio of the image was much improved. Stokes parameters of the probe were evaluated, from which suitable compensation for polarization transmission was performed. This provides a possibility of obtaining quantitative evaluation of magneto-optical parameters in the submicrometer region. A Clear MO image of recorded marks on a $\mathrm{Pt} / \mathrm{Co}$ disk was obtained with $100 \mathrm{~nm}$ resolution.

Key words: scanning near-field microscope, magnetooptical imaging, sub-micrometer region, $\mathrm{P} t / \mathrm{Co}$ magnetooptical disk,

\section{Introduction}

Scanning near-field optical microscopes (SNOM) have been attracting interest for use in magneto-optical (MO) imaging of small magnetic structures with subwavelength dimensions.[1-4] These works employ straight fibers and lateral shear force feedback. Polarization transmission properties of the fiber probes were generally poor, so that $\mathrm{MO}$ images with a high contrast ratio were not obtained. In order to overcome the drawback Kottler et al. employed the fiber probe only to detect the intensity of the light in the detection mode. They detected MO signal as a difference in intensities between the right and left circularly polarized light beams. [5]

We have been developing a SNOM system employing a bent optical fiber probe in the illumination mode, the probe being operated as an AFM cantilever, and have succeeded in MO imaging of recorded marks on a bismuth-substituted dysprosium iron garnet $\mathrm{MO}$ disk using the crossed polarizer method as described previously. $[6,7]$ We thus confirmed that the light polarization can be transmitted even in the near-field

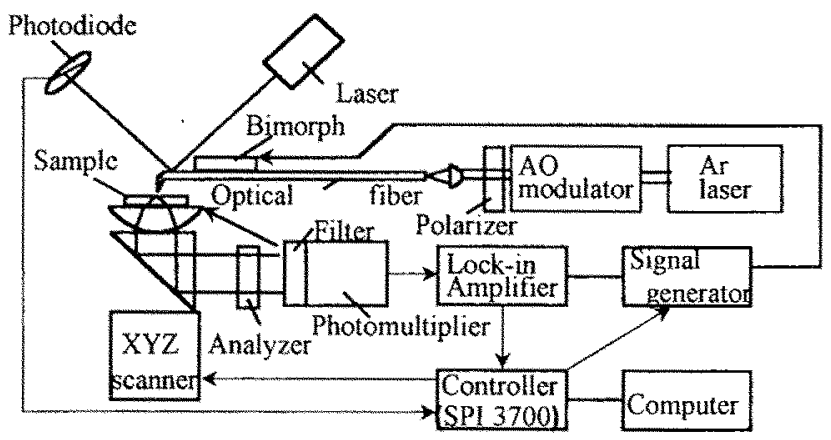

Fig.1 A schematic diagram of SNOM/AFM system for $\mathrm{MO}$ imaging using crossed polarizer technique. regime if appropriate fiber probes are adopted. However, improvement of the quality of the polarization image was strongly needed since the contrast ratio of the $\mathrm{MO}$ SNOM image in this system was as low as 0.1 .

For this purpose a polarization modulation technique using a photo-elastic modulator (PEM) was exploited. [8] This leads to an improvement of the contrast ratio to a value as high as 0.6 . Nevertheless quantitative values of magneto-optical parameter was still difficult to obtain since the rotation and ellipticity were often mixed due to complicated the polarization-transmission properties of the fiber probe.

Quantitative data of polarization transmission of the fiber probe are necessary in order to compensate the modification caused by the probe and get a clear image. For this purpose we determined Stokes parameters. The result shows that the optical properties of carefully prepared fiber probes are quite simple; they can be treated as if they were a wave-plate and can be compensated by optical elements.

\section{Experimental Details of SNOM/AFM System}

A schematic diagram of the SNOM/AFM system employed in this study is illustrated in Fig. 1. Our SNOM for MO imaging is based on a commercially available AFM unit (model SPI3700, Seiko Instruments). An optical probe mounted on a bimorph is vibrated vertically against the sample surface at its resonant frequency (typically $10-40 \mathrm{kHz}$ ). A laser reflected on a polished surface of the probe is detected, and the change of the vibrational amplitude of the probe is monitored. The tip-sample separation is controlled by maintaining the amplitude at a constant value. The vibration amplitude is varied at $10-100 \mathrm{~nm}$ by a voltage applied to the bimorph. Figure 2 shows an SEM image of the bent optical fiber probe employed. A single-mode optical fiber whose core and cladding is 3.2 and $125 \mu \mathrm{m}$ in diameter, respectively, is pulled to form the tip and bent by the irradiation of a $\mathrm{CO}_{2}$ laser. The bent angle is about $80^{\circ}$, and the point is $0.5 \mathrm{~mm}$ away from the apex of the tip. By coating the side of the tapered probe with a $100-$

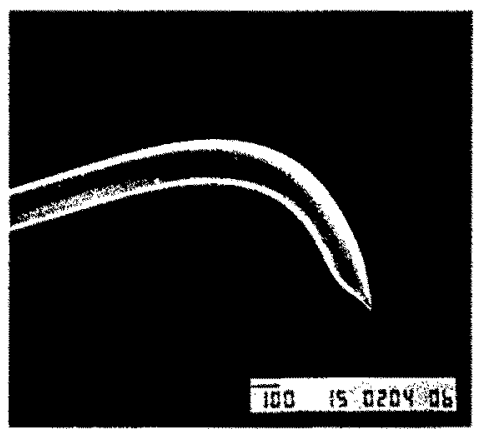

Fig. 2 Bent fiber probe used as an AFM cantilever 
$150 \mathrm{~nm}$ thick aluminum film, an aperture is fabricated at the apex of the probe. The aperture is $80-100 \mathrm{~nm}$ in diameter. As stated above the probe works as a cantilever for AFM operation, so that simultaneous observation of topographic and $\mathrm{MO}$ images is possible. A 488-nm $\mathrm{Ar}^{*}$ ion laser with an output power of $50 \mathrm{~mW}$ is used as the light source. The beam is modulated by an acousto-optic (AO) modulator to improve the $S / N$ ratio of the signal through lock-in detection. The light is passed through a polarizer and focused into the optical fiber probe using collimating optics. The evanescent light field emerging from the aperture of the probe is incident upon the surface of the sample. The transmitted light goes through the sample and is detected by a photomultiplier through a collimating lens, a dichroic mirror, an analyzer and an optical filter. Details of the system were described elsewhere. $[6,7]$

\section{Observation of Recorded Marks by Crossed Polarizer Technique}

Using the SNOM/AFM system with the crossed polarizer technique, magneto-optically recorded marks on a bismuth-substituted dysprosium iron garnet (DIG) disk were observed as shown in Fig. 1. The film was deposited on a glass substrate and had a thickness of 100 $\mathrm{nm}$. The Faraday rotation angle of the garnet film was evaluated to be $1.7^{\circ}$ by means of far-field optics. The marks were recorded by light intensity modulation using a conventional MO read-write system with a focused laser beam. The scan area was (a) $15 \times 15 \mu \mathrm{m}^{2}$ and (b) $2 \times 2 \mu \mathrm{m}^{2}$. Small circular marks $0.7 \mu \mathrm{m}$ in diameter were clearly observed. Resolution of the MO image was slightly beyond the diffraction limit. Improvement of signal to noise ratio (SNR) is necessary to obtain higher resolution.

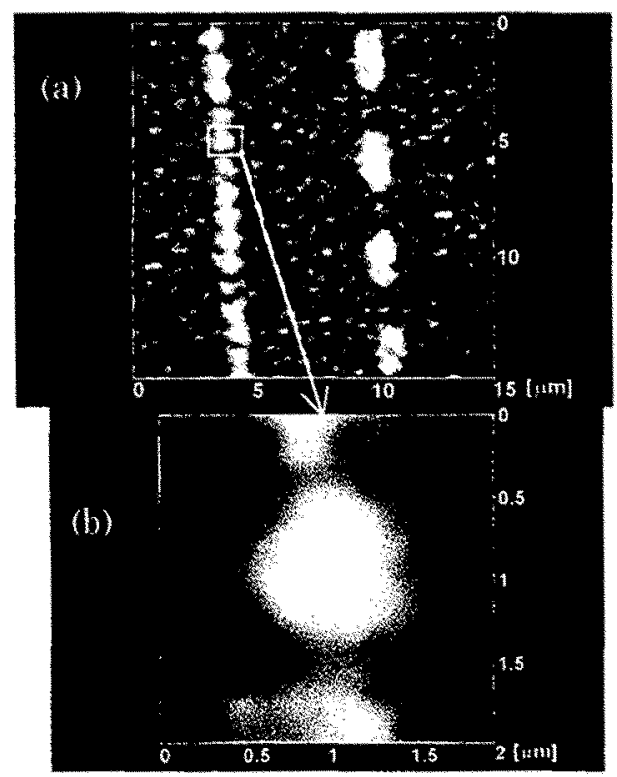

Fig. $3 \mathrm{MO}-\mathrm{SNOM}$ image of recorded marks on $\mathrm{Bi}$ substituted DIG film by a cross-polarizer method;

(a) wide area scan, (b) narrow area scan.

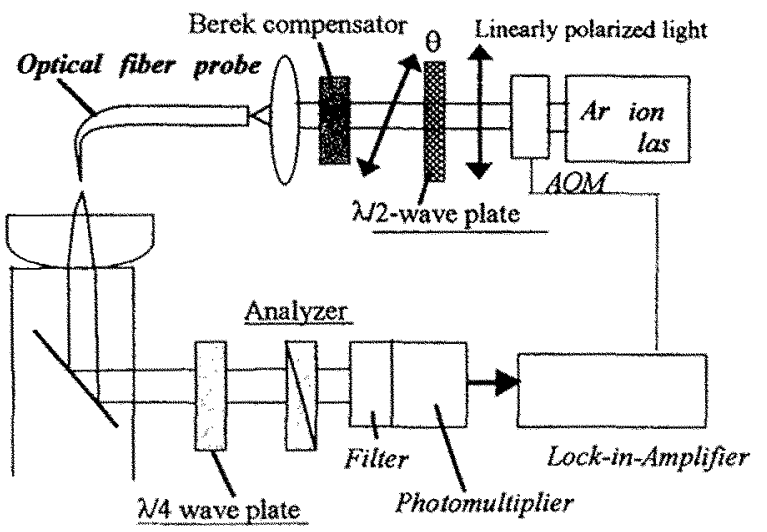

Fig. 4 A schematic diagram of a measuring system for Stokes parameters

\section{Evaluation of Stokes Parameters in Optical Fiber Probes}

Quantitative knowledge of polarization states of the light emerging from the aperture in the probe is indispensable in order to obtain suitable transmission properties of the probe for MO use. We measured the Stokes parameters to evaluate the polarization transmission characteristics.

The Stokes parameters are given by

$$
\begin{aligned}
& S_{0}=I_{x}+I_{y y} \\
& S_{1}=I_{x}-I_{y} \\
& S_{2}=2 I_{x y}-S_{0 x} \\
& S_{3}=2 I_{q x y}-S_{0 y}
\end{aligned}
$$

where $I_{x}, I_{x y}$ and $I_{y}$ are intensities measured with an analyzer having angles of $0^{\circ}$ (x-axis), $45^{\circ}$ and $90^{\circ}(y-$ axis), respectively, and $I_{\alpha x y}$ is that with a quarter wave plate. Therefore, $S_{0}, S_{1}, S_{2}$ and $S_{3}$ represent intensities of the light, the polarized light along $\mathrm{X}$-axis, the polarized light along $45^{\circ}$ and the circularly polarized light, respectively. The degree of polarization $P$ is given by

$$
\mathrm{P}=\left(\mathrm{S}_{1}{ }^{2}+\mathrm{S}_{2}{ }^{2}+\mathrm{S}_{3}{ }^{2}\right)^{1 / 2} / \mathrm{S}_{0} \text {. }
$$

Figure 4 shows a schematic diagram of a measuring system for the Stokes parameters. Light with the wavelength of $488 \mathrm{~nm}$ from an $\mathrm{Ar}^{+}$ion laser is modulated by an AOM to carry out the lock-in detection, and coupled to an optical fiber probe. The polarization property of the light emitted from the aperture was measured in the far-field region without samples to obtain the polarization effect of the fiber probe separately, avoiding any interaction between the aperture and samples. A half-wave $(\lambda / 2)$ plate, a quarter-wave $(\lambda / 4)$ plate and an analyzer are inserted into the optical path to determine the Stokes parameters. In order to compensate the anisotropic polarization state of the beam emitted from the probe, a Berek compensator is placed in front of the fiber coupler. Details of the measurements will appear elsewhere.[11] 


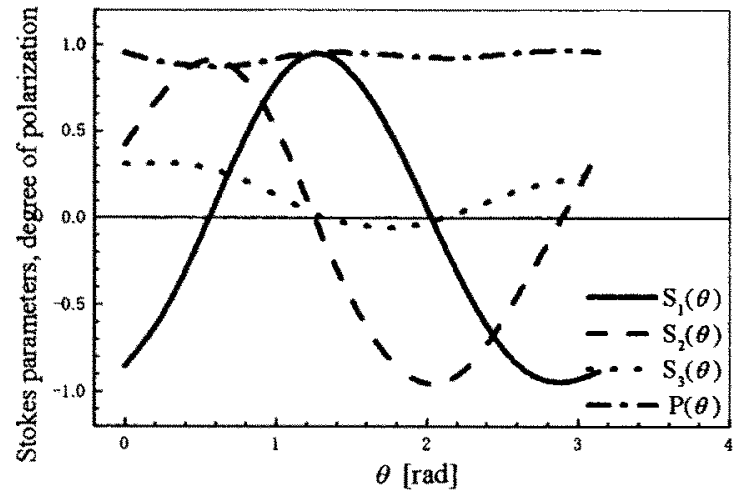

Fig. 5(a) Polarization properties of a bent fiber without tapering expressed in terms of Stokes parameters

In Fig. 5 are shown the Stokes parameters normalized by $S_{0}$ together with the degree of polarization as a function of the polarizing angle $\theta$ for (a) a bent optical fiber without tapering and (b) an optical fiber probe fabricated by bending, tapering and metal-coating. In the case of ideal linearly polarized light, $S_{1}$ and $S_{2}$ vary as a sinusoidal curve with the rotation angle, while $S_{3}$ is zero for all angles. On the other hand, as illustrated in Figs 5 (a) and $5(\mathrm{~b}), S_{3}$, as well as $S_{1}$ and $S_{2}$, show a sinusoidal variation against $\theta$. It is found that an optical anisotropy is obviously induced by bending. This result indicates that the bent optical fiber and the bent optical probe act as if it were a wave plate as we have reported previously, $[6,7]$ although the cause of the difference in the polarization properties between the bent optical fiber and the bent optical fiber probe are not cear. Furthermore, it is surprising that the degree of the polarization is almost maintained after transmission from the aperture of a bent optical fiber probe.

We found that a carefully prepared probe works as if it were a wave-plate. Therefore the polarization transmission properties can be compensated by a suitable compensator. Figure 6 shows Stokes parameters of bent optical fiber probe compensated by the Berek compensator placed in front of the fiber coupler. The amplitude of $S_{3}$ is reduced to $<0.1$ while $S_{1}$ and $S_{2}$ vary sinusoidally.

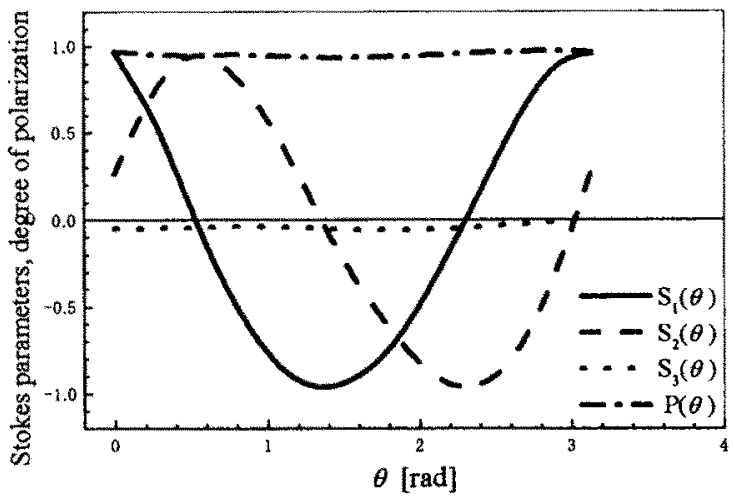

Fig.6 Polarization properties of a fiber probe after compensation.

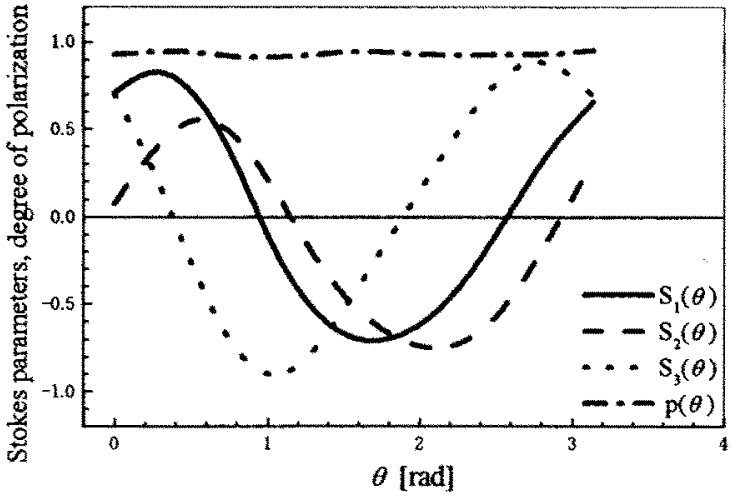

Fig. 5(b) Polarization properties of a bent and tapered fiber probe expressed in terms of Stokes parameters

\section{MO-SNOM System with Polarization Modulation}

In order to improve the SNR, we adopted a polarization modulation technique using a photoelastic modulator (PEM). The technique has been used for MO spectroscopy and has been proved to be a powerful tool for the measurement of small MO signals. $[9,10] \mathrm{A}$ schematic diagram of this system is given in Fig. 7. Using this technique, the MO signals can be obtained by detecting intensities of light modulated by the PEM modulated at a frequency $p$. In principle, the $p$ and $2 p$ components of the detected signals correspond to the Faraday ellipticity and the Faraday rotation, respectively, if ideal polarization properties are maintained during transmission through the fiber probe. The Faraday ellipticity and the Faraday rotation are given by

$$
\begin{aligned}
& \eta_{\mathrm{F}}=I(p) /\left\{I(0) \cdot 4 J_{1}\left(\delta_{0}\right)\right\} \cdot C_{\eta}, \\
& \theta_{\mathrm{F}}=-I(2 p) /\left\{I(0) \cdot 4 J_{2}\left(\delta_{0}\right)\right\} \cdot C_{\theta},
\end{aligned}
$$

where $I(0)$ is the intensity of the light, $J_{\mathrm{n}}$ is a Bessel function of the $n$-th order and $\delta_{0}$ is the amplitude of retardation by the PEM. In the measurement, these values should be corrected by $C_{\eta}$ and $C_{\theta}$, which are factors affected by experimental setups such as optical elements or electric devices

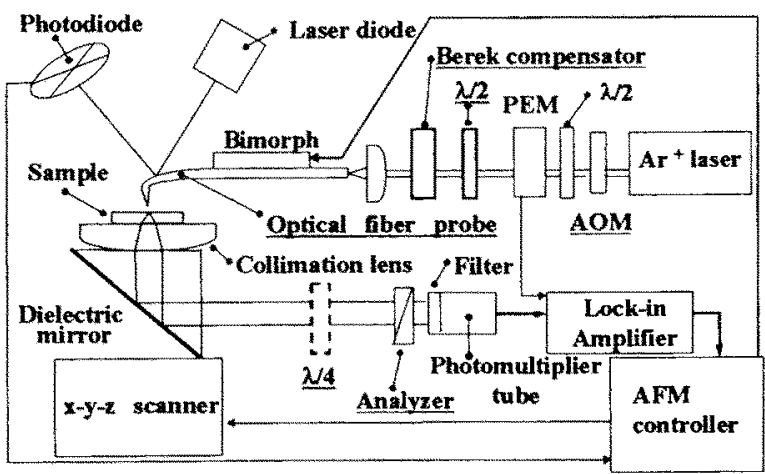

Fig. 7 A schematic diagram of MO-SNOM using polarization modulation technique. 


\section{Observation of Recorded Marks on a Pt/Co Disk}

Using the newly designed SNOM system we observed magneto-optically recorded marks on a $\mathrm{Pt} / \mathrm{Co}$ disk. The disk consists of a Pt $(0.8 \mathrm{~nm}) / \mathrm{Co}(0.3 \mathrm{~nm})$ multilayer whose total thickness is $15 \mathrm{~nm}$, a $\mathrm{Pt}(3 \mathrm{~nm})$ buffer, and a SiN/glass substrate. The marks were recorded by the magnetic-field modulation method with a length of $10 \mu \mathrm{m}$ and a width of $1 \mu \mathrm{m}$, which leads to crescent shaped recorded mark patterns. Magneto-optical spectra of the Pt/Co multilayer were measured using a magneto-optical spectrometer, from which the Faraday rotation and Faraday ellipticity at $488 \mathrm{~nm}$ were determined to be $0.74^{\circ}$ and $0.47^{\circ}$, respectively. It is very hard to get a good image without a modulation technique.

Figure 8 shows a topographic (AFM) image and an MO image of a portion of the Pt/Co disk, in which grooves are partly observed. As shown in Fig. 8 the MO image suffers an artifact due to the topographic structure in the grooved portion, whereas only images of magnetooptical origin are clearly resolved in the groove-free portion. Figure 9 shows magneto-optical images of a recorded mark observed in the groove-free portion of the $\mathrm{Pu}$ Co MO disk visualized by (a) the $p$ component and (b) the $2 p$ component of the detected signals, of which the contrast is proportional to Faraday ellipticity $\eta_{\mathrm{F}}$ and Faraday rotation $\theta_{\text {}}$, respectively. Recorded marks having a crescent-like shape characteristic of the magnetic field modulation method are clearly resolved. A resolution of approximately $100-150 \mathrm{~nm}$ was obtained from a line scan on the MO image.

Signal outputs, which are differences in voltages between a recorded and an unrecorded area, were obtained to be $I(p)=75$ and $I(2 p)=90 \mathrm{mV}$ for $\eta_{\mathrm{F}}$ and $\theta_{\mathrm{F}}$, respectively. Considering the factors $C_{\eta}$ and $C_{\theta}, \eta_{\mathrm{F}}$ and $\theta_{\mathrm{F}}$ can be obtained as $288 / /(0)$ and $692 / /(0)$, respectively. The relative magnitude is consistent with the rotation angles of $\eta_{k}$ and $Q_{k}$ measured by conventional Faraday spectroscopy. We are convinced that the evaluation of quantitative values in MO imaging can be achieved.

\section{Conclusion}

An MO-SNOM system was developed based on a conventional AFM imaging system. Topographic and MO images were simultaneously obtained by using a bent-type optical fiber probe operated as a cantilever of an AFM system. The Stokes parameters were measured to understand the polarization properties of the optical fiber probe. It was found that the probe acts as if it were a wave plate, and the degree of polarization is maintained after emission from the probe. The retardation induced by the probe was evaluated in terms of the Stokes parameter $S_{3 \text {, }}$ and was successfully compensated by using a Berek compensator. We believe that this method can contribute to the study of magnetism in the subwavelength scale.

\section{Acknowledgments}

This work has been supported in part by a Grant-inAid for Scientific Research from the Ministry of Education, Science, Sports and Culture, The authors

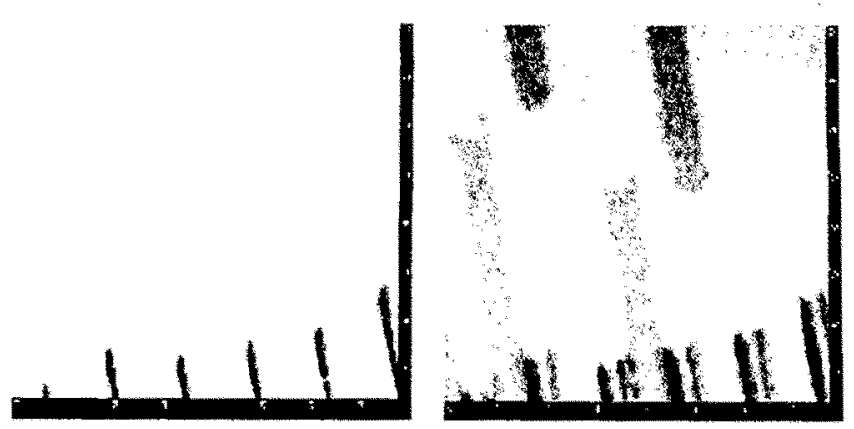

Fig.8 Topographic image (lef) and Magneto-optical image (right) observed by scanning near-field magnetooptical microscope
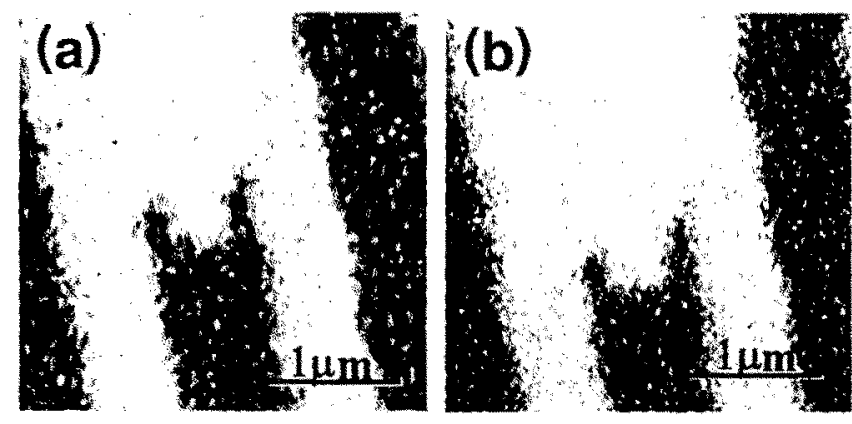

Fig. 9 MO images of marks on Pt/Co disk recorded by the light pulse magnetic field modulation method observed by MO-SNOM, (a) the $p$ component, (b) the $2 p$ component, corresponding to Faraday ellipticity and Faraday rotation, respectively.

wish to thank Mr. N. Kawamura of NHK Science and Technical Research Laboratory for supplying the garnet disk. They are also indebted to Dr. J. Ushiyama of Hitachi Ltd, Central Research Laboratory and Dr. $\mathrm{H}$. Awano of Hitachi Maxell Ltd. for supplying a Pt/Co disk with $\mathrm{MO}$ recorded marks.

\section{References}

[1] E. Betzig, J.K.Trautman, T.D. Harris, J.S. Weiner, R.L. Kostelak: Science 251 (1991) 1468.

[2] J.K. Trautman, E. Betzig, J.S. Weiner, D.J. DiGiovanni, T.D. Harris, F. Hellman, E.M. Gyorgy: J. Appl. Phys. 71 (1992) 4659

[3] E. Betzig, J.K. Trautman, J.S. Wiener, T.D. Harris, R. Wolfe: Appl. Opt. 31 (1992) 4563.

[4] E. Betzig, J.K. Trautman, R. Wolfe, E.M. Gyorgy, P.L. Finn, M.H. Kryder, C.H. Chang: Appl. Phys. Lett. 61 (1992) 142

[5] V. Kottler, N. Essaidi, N. Ronarch, C. Chappert and Y. Chen: J. Magn. Magn. Mater. 165,1 (1997)

[6] Y. Mitsuoka, K. Nakajima, K. Homma, N. Chiba, H. Muramatsu, T. Ataka, K. Sato: J. Appl. Phys. 83 (1998) 3998

[7] K. Nakajima, Y. Mitsuoka, N. Chiba, H. Muramatsu, T. Ataka, K. Sato, M. Fujihira: Ultramicroscopy 71 (1998) 257.

[8] K. Sato, S. Toya, Y. Mitsuoka and K. Nakajima: Digest of 15 th Int. Colloq. Magn. Films \& Surfaces, Sunshine Coast, Australia, August 4-8, 1997 pp.134-135.

[9] K. Sato, Jpn. J. Appl. Phys. 20, 2403, 1981 .

[10] K. Sato, H. Hongu, H. Ikekame, Y. Tosaka, M. Watanabe, K. Takanashi and H. Fujimori, Jpn. J. Appl. Phys. 32, 989 (1993)

[11] T. Ishibashi, T. Yoshida, A. Ijima, K. Sato, Y. Mitsuoka and K. Nakajima: J. Microscopy (to be published) 